Draft Version OCtOBER 30, 2018

Preprint typeset using $\mathrm{IAT}_{\mathrm{E}} \mathrm{X}$ style emulateapj v. 03/07/07

\title{
INFERRING PHYSICAL PARAMETERS OF COMPACT STARS FROM THEIR F-MODE GRAVITATIONAL WAVE SIGNALS
}

\author{
H. K. LAU* ${ }^{*}$ P. T. LEUNG ${ }^{\dagger}$ AND L. M. LiN ${ }^{\ddagger}$ \\ Department of Physics and Institute of Theoretical Physics, \\ The Chinese University of Hong Kong, Shatin, Hong Kong SAR, China.
}

(Dated: October 30, 2018)

Draft version October 30, 2018

\begin{abstract}
We propose here a robust scheme to infer the physical parameters of compact stars from their $\mathrm{f}$ mode gravitational wave signals. We first show that the frequency and the damping rate of $\mathrm{f}$-mode oscillation of compact stars can be expressed in terms of universal functions of stellar mass and moment of inertia. By employing the universality in the f-mode one can then infer accurate values of the mass, the moment of inertia and the radius of a compact star. In addition, we demonstrate that our new scheme works well for both realistic neutron stars and quark stars, and hence provides a unifying way to infer the physical parameters of compact stars.

Subject headings: gravitational waves — stars: neutron — stars: oscillations (including pulsations) equation of state - relativity
\end{abstract}

\section{INTRODUCTION}

Ever since the first prediction of neutron stars (NS) by Baade \& Zwicky (1934), NS have become a major test bed of various theories of dense matter and nuclear matter. The extreme high pressure and density achievable inside NS offers an ideal and unique environment to infer the equation of state (EOS) of nuclear matter from astronomical observations. On the other hand, the possible existence of quark stars (QS) would provide a strong support to a new form of matter, namely, the quark matter (Itoh 1970, Bodmer 1971, Witten 1984). Moreover, the size and the mass of QS could also cast light on the properties of quark matter. Thus, extracting useful information and constraints about nuclear and particle physics from astronomical observations of compact stars like NS and QS has become an active direction in astrophysics (see, e.g., Lattimer \& Prakash 2007, Haensel et al. 2007, for reviews).

Since the density in the core of a NS (or QS) can be several times the normal nuclear density, the EOS there is rather uncertain. Various physical phenomena including pion condensation, hyperon formation and deconfined quark matter have been considered by theoretical nuclear physicists and sophisticated techniques of manybody calculations have been developed to yield a bunch of EOS intended for the description of the deep interior of compact stars. It is standard practice for astrophysicists to construct compact stars with these EOS for nuclear matter and establish various relationships among different physical quantities which could be obtained from astronomical observations. Motivated by the possibilities of constraining EOS of nuclear matter and extracting physical parameters of compact stars as well, researchers in astrophysics and nuclear physics have been actively seeking for EOS-dependent and EOS-independent

* Present address: Department of Physics, University of Toronto, 60 St. George St., Toronto, M5S 1A7, Ontario, Canada; email: kero.lau@utoronto.ca

†email: ptleung@phy.cuhk.edu.hk

‡email: lmlin@phy.cuhk.edu.hk relationships by examining the physical characteristics of compact stars constructed with different EOS (see, e.g., Lattimer \& Prakash 2001, Beiger \& Haensel 2002, Lattimer \& Schutz 2005, Beiger et al. 2005, Lattimer \& Prakash 2007, Haensel et al. 2007). For example, Lattimer \& Prakash (2001) compared the structure of compact stars of various kinds and discovered several empirical relationships connecting different physical characteristics of a star, such as the mass, the radius, the moment of inertia, and the mass distribution function as well. Such relationships can be applied to infer the physical attributes of a compact star, including its EOS, from astronomical observations.

The main objective of the present paper is to unveil the universality embedded in the pulsation frequencies of non-rotating compact stars and to exploit these findings to infer the physical characteristics of NS or QS (e.g., mass, radius, moment of inertia and EOS) from their oscillation spectra. The pulsations of compact stars are an interesting and popular topic in its own right, for gravitational waves $(\mathrm{GW})$ can be generated in such processes (see Thorne \& Campolattaro 1967, for a seminal exposition of the topic). It is indisputable that the detection of GW would be a major milestone in general relativity and astrophysics. Several Earth-based GW interferometric detectors such as LIGO, VIRGO, GEO600 and TAMA300 have been operating. While the current detectors are still not sensitive enough to detect GW directly, interesting upper limits have been placed on either the GW strains or event rate for several potential astrophysical sources (see, e.g., Abbott et al. 2009a.b.c, for the results obtained from the latest science runs of LIGO).

Owing to the energy carried away by GW, pulsations of compact stars are damped harmonic oscillations, which are analyzed in terms of quasi-normal modes (QNM) (see, e.g. Press 1971, Leaver 1986, Ching et al. 1998, Kokkotas \& Schmidt 1999). Each QNM is characterized by a complex eigenfrequency $\omega=\omega_{\mathrm{r}}+i \omega_{\mathrm{i}}$ and has a time dependence $\exp (i \omega t)$, displaying exponential decay with a damping time $\tau \equiv 1 / \omega_{\mathrm{i}}$. Several attempts have been made to relate $\omega_{\mathrm{r}}$ and $\omega_{\mathrm{i}}$ (or $\tau$ ) 
to the mass $M$ and the radius $R$ of a compact star and some universal relationships which are, to certain degree of accuracy, EOS-independent have been found (Andersson \& Kokkotas 1996, 1998, Benhar et al. 1999, 2004, Tsui \& Leung 2005b). However, most of these relationships fail to describe QNM of QS, which are stiff and self-bound.

On the other hand, Lattimer \& Schutz (2005) found an empirical relation approximately expressing the moment of inertia in terms of the mass and the radius. Such a relationship is universal as long as the EOS under consideration is not too soft (e.g., hyperon matter) or too stiff (e.g., quark matter). They proposed to use the universality to estimate the radius of a compact star from its mass and moment of inertia, with the latter two quantities being measurable for double pulsar systems by considering the spin-orbit coupling effect in general relativity (Lattimer \& Schutz 2005).

Motivated by the discovery of Lattimer \& Schutz (2005), we propose here to investigate the correspondence between the QNM frequency (both $\omega_{\mathrm{r}}$ and $\omega_{\mathrm{i}}$ ) of the (fundamental) f-mode oscillations, $M$ and the moment of inertia $I$ of compact stars. We show that there exist EOS-independent universal relationships between them, namely equations (6) and (7), which are accurate up to a few percent or better and work nicely for QS as well. Based on equations (6) and (7), a feasible scheme is in turn established to determine the mass and the moment of inertia of a compact star once its f-mode frequency is found from GW observations. As the radius $R$ of a NS (or QS) is approximately expressible in terms of the mass and the moment of inertia, our scheme can also yield good estimate of the radius and hence imposing constraints on the EOS (Bejger \& Haensel 2002, Lattimer \& Schutz 2005).

The outline of the paper is as follows. Section 2 is a brief review on the the behavior of f-mode oscillations of compact stars (Tsui \& Leung 2005b) and the universal relationships among the mass, the radius and the moment of inertia of compact stars (Beiger \& Haensel 2002, Lattimer \& Schutz 2005). In Section 3 we introduce an accurate universal relation between the scaled frequency $M \omega$ and the physical quantity $\sqrt{M^{3} / I}$. In Section 4 we establish a feasible and robust scheme to apply our findings reported in Section 3 to invert the mass, the moment of inertia, and in turn the radius of a compact star if its f-mode GW signal is detected. We finally summarize our paper in Section 5 with a brief discussion. Unless otherwise noted, we adopt geometric units with $G=c=1$ throughout the whole paper, and use kilometers as the unit of lengths.

\section{GW AND MOMENT OF INERTIA OF COMPACT STARS}

As mentioned in Section 1, the oscillation modes of compact stars are damped due to the emission of GW. Hence, they are called quasi-normal modes, which can be further divided into polar and axial classes in each angular momentum sector according to the property of relevant metric and fluid perturbations under inversion. For typical non-rotating compact stars, the polar class includes (fundamental) f-mode, (gravity) g-mode, and (pressure) p-mode and (spacetime) w-mode, while for non-rotating perfect fluid stars the axial class contains only the w-modes (see, e.g., Kokkotas \& Schmidt 1999, for a review on the property of these modes). The methods for evaluation of QNM of compact stars are well documented in several papers and reviews (see, e.g., Lindblom \& Detweiler 1983, Detweiler \& Lindblom 1985, Chandrasekhar \& Ferrari 1991, Kokkotas \& Schmidt 1999).

The study of QNM of compact stars has a long history dating back to the pioneering work of Thorne \& Campolattaro (1967). It is by now well established that the study of GW mode spectra of compact stars could provide useful information about the internal structure of the stars (see, e.g., Andersson \& Kokkotas 1996, 1998, Kokkotas et al. 2001, Benhar et al. 1999, 2004, Tsui \& Leung 2005a, b, Sotani \& Harada 2003, Sotani et al. 2004, Wong et al. 2009). As it is expected that the direct detection of GW will be realized in the near future, different proposals have been put forward to determine the physical parameters (e.g., mass, radius and EOS) of a compact star from its GW spectra. In the present paper we focus our attention on an EOSindependent universal behavior of the quadrupolar (angular momentum index $l=2$ ) f-mode and show that such universality can lead to accurate determination of the mass and the moment of inertia of a compact star (NS or QS). Combining our results with equations (3), (4) and (5), one can also find an approximate value of the radius.

The reason why we place special emphasis on the fmode is that the relatively low frequency $(\sim 1-3 \mathrm{kHz})$ of the f-mode makes it more promising to be detectable by GW detectors than other modes. For example, considering a compact star $10 \mathrm{kpc}$ from us, Kokkotas et al. (2001) estimated that the energy required in the f-mode in order to lead to a detection with signal-to-noise ratio of 10 by the LIGO detector is $4.9 \times 10^{-5} M_{\odot} c^{2}$. For comparison, in order to achieve the same signal-to-noise ratio, the energy in the p-mode (w-mode) must be $4.0 \times 10^{-3} M_{\odot} c^{2}$ $\left(6.8 \times 10^{-2} M_{\odot} c^{2}\right)$. The energy requirement is much relaxed for the advanced LIGO detector and the same signal-to-noise ratio is achieved if the energy in the fmode is $8.7 \times 10^{-7} M_{\odot} c^{2}$. Since the total energy released in violent astronomical events such as supernova explosions could be as much as $10^{-2} M_{\odot} c^{2}$ in some optimistic estimates (Kokkotas et al. 2001), it seems plausible that the f-mode QNM signal of a fiercely pulsating compact star inside our galaxy could be detected by future GW detectors, especially when the narrow-banding capability is available to enhance the sensitivity of the detectors in the high-frequency region at around $1 \mathrm{kHz}$.

There have been several attempts to search for an approximately EOS-independent behavior of the f-mode (Andersson \& Kokkotas 1996, 1998, Benhar et al. 1999, 2004, Tsui \& Leung 2005b). In particular, Tsui \& Leung (2005b) showed that the scaled frequency $M \omega$ for the $\mathrm{f}-$ mode of realistic NS is essentially a universal function of the compactness $M / R$, which can be approximated by the quadratic formulae:

$$
\begin{aligned}
& M \omega_{\mathrm{r}}=0.15\left(\frac{M}{R}\right)^{2}+0.56\left(\frac{M}{R}\right)-0.02, \\
& \frac{M \omega_{\mathrm{i}}}{10^{-4}}=-5.8\left(\frac{M}{R}\right)^{2}+6.7\left(\frac{M}{R}\right)-0.62 .
\end{aligned}
$$


These two formulae are good approximation for most NS. However, the f-modes of QS do not follow equations (11) and (2). Instead, they are approximated better by another quadratic fit. The difference between the behaviors of the f-modes of NS and QS are expected because the density profile of a QS is nearly uniform, which is very different from that of a NS.

On the other hand, Beiger \& Haensel (2002) and Lattimer \& Schutz (2005) discovered some empirical relationships relating $I$ and compactness $M / R$. For NS, Beiger \& Haensel (2002) found that

$$
\tilde{I}= \begin{cases}x /(0.1+2 x) & \text { if } x \leq 0.1 \\ 2(1+5 x) / 9 & \text { if } x>0.1\end{cases}
$$

where the scaled moment of inertia $\tilde{I}=I / M R^{2}$ and $x=\left(M / M_{\odot}\right)(\mathrm{km} / R)$. Lattimer \& Schutz (2005) also proposed an alternative expression:

$$
\tilde{I}=0.237\left(1+4.2 x+90 x^{4}\right),
$$

which yields better agreement for NS with higher compactness. However, both equations (3) and (4) fail to describe the case of QS. Instead, Beiger \& Haensel (2002) found another relationship for QS:

$$
\tilde{I}=\frac{2}{5}(1+x) \text {. }
$$

The distinction between the behaviors of NS and QS is also expected and attributable to the difference in the stiffness between nuclear and quark matters.

Assuming that both $I$ and $M$ are obtained from observations, Lattimer \& Schutz (2005) further argued that the radius $R$ can be found by solving these equation. However, two remarks about this method are in order. First, for the NS case the data points corresponding to stars characterized by different EOS are rather scattered and may deviate appreciably from equations (3) and (4) - As a consequence, the value of $R$ obtained in this way is subjected to uncertainties depending on the EOS. Second, additional information has to be gathered to distinguish between the data of QS and NS beforehand. Regardless of these remarks, it is observed that for a fixed EOS the scaled moment of inertia increases monotonically with the compactness, indicating a one-one correspondence between these two quantities. In the following discussion we shall consider the feasibility to replace the compactness used in equations (1) and (2) with the moment of inertia.

\section{UNIVERSALITY IN F-MODE OSCILLATIONS}

It is interesting to note from the reviews sketched in Sections 2 that both the scaled moment of inertia $I / M R^{2}$ and scaled f-mode frequency $M \omega$ are rather insensitive to EOS and are, within certain accuracy, universal functions of compactness (Bejger \& Haensel 2002, Lattimer \& Schutz 2005, Tsui \& Leung 2005b). Such coincidence is interesting, reflecting the fact that compactness is a useful parameter characterizing a compact star. However, it is also noticed that the scaled f-mode frequencies (or the scaled moments of inertia) of NS and QS follow two different universal behaviors. The disparity in the universal behaviors of NS and QS is attributable to the difference in their density profiles.

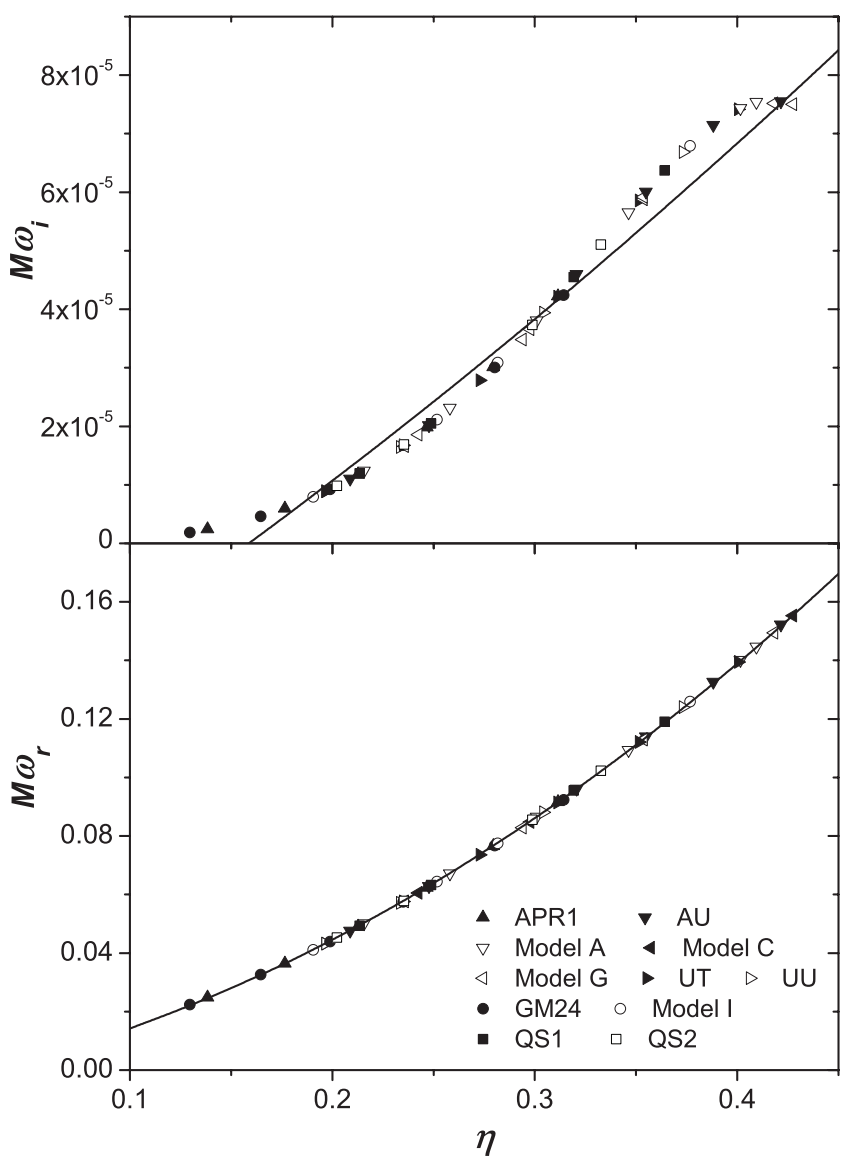

FIG. 1. - The scaled frequency of f-mode QNM is plotted against the effective compactness $\eta$. The solid lines in the lower and upper panels respectively represent the best quadratic fits in $\eta$ to $M \omega_{\mathrm{r}}$ and $M \omega_{i}$. In particular, the solid line in the lower panel is given by equation (6).

In the present paper we propose to examine the possibility of using the moment of inertia as an independent parameter, instead of the compactness, to characterize the f-mode spectra and in turn to search for any universality underlying these spectra. The physical motivation of our proposal is that the moment of inertia carries richer information about the mass distribution inside a star, which certainly affects f-mode oscillations. Hence, the relationship between the moment of inertia and the fmode is likely to be more direct and less EOS-dependent. Besides, as mentioned in Section 2, for compact stars with a fixed EOS, the moment of inertia bears a one-one correspondence with the compactness. To this end, we replace the compactness $M / R$ by another dimensionless factor $\eta \equiv \sqrt{M^{3} / I}$, which is proportional to the compactness for stars with uniform densities and is termed as the effective compactness.

The scaled f-mode frequencies for various realistic NS and QS are plotted against the effective compactness $\eta$ in Figure 1, where the f-mode QNM frequencies of the compact stars constructed with the following EOS are shown:

1. Nine popular ordinary nuclear matter EOS for NS, including Model A (Pandharipande 1971a); Model C (Pandharipande 1971b); Model G (Canuto \& Chitre 1974); Model I (Cohen et al. 
TABLE 1

Percentage error of Different universal formulae

\begin{tabular}{ccccc}
\hline quantity & equation & $\sigma_{1}$ & $\sigma_{2}$ & $\sigma_{3}$ \\
\hline \hline$\omega_{\mathrm{r}}$ & $(1)$ & 5.8 & 4.2 & 21 \\
\hline$\omega_{\mathrm{r}}$ & $(\underline{6})$ & 0.37 & 0.56 & 0.70 \\
\hline \hline$\omega_{\mathrm{i}}$ & $(\underline{2})$ & 220 & 6.1 & 55 \\
\hline$\omega_{\mathrm{i}}$ & $(\underline{7})$ & 1.5 & 2.1 & 1.1 \\
\hline
\end{tabular}

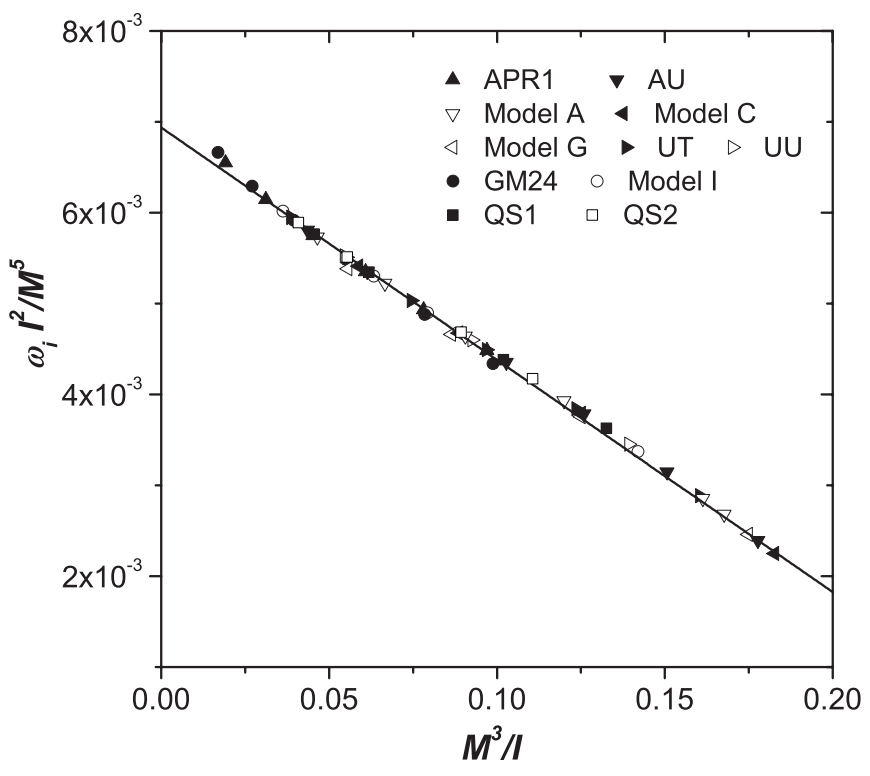

FIG. 2.- $\omega_{\mathrm{i}} I^{2} / M^{5}$ is plotted against $\eta^{2}$ (i.e., $M^{3} / I$ ). The solid line is the best linear fit to the distribution (see equation (7)).

1970); APR 1 (Akmal et al. 1998); AU, UU and UT (Wiringa et al. 1988); and GM24 (Glendenning 1997, p. 244), which cover a wide range of softness (with an adiabatic index $\gamma \sim 1.6-4$ in the highdensity region).

2. Two QS models, QS1 and QS2, constructed with the MIT bag model where the effects of strange quark mass and temperature are considered (see, e.g., Glendenning 1997). Characteristic physical parameters for QS1 (QS2) are as follows: the bag constant $B$ is given by $B^{1 / 4}=$ 154.5 MeV (150.0 MeV), strange quark mass $m_{s}=$ $150 \mathrm{MeV}(100 \mathrm{MeV})$, and temperature $T=0 \mathrm{MeV}$ $(10 \mathrm{MeV})$.

It is clearly seen that the data for NS and QS now display almost identical universal behaviors with high degree of accuracy. In fact, a quadratic expression in $\eta$ of the form

$$
M \omega_{\mathrm{r}}=-0.0047+0.133 \eta+0.0575 \eta^{2},
$$

suffices to yield a perfect fit to $M \omega_{\mathrm{r}}$ of all stars (NS and QS). On the other hand, despite the fact that $M \omega_{i}$ of all stars fall on a common smooth curve, quadratic fits in $\eta$ do not work very well in this case.

Motivated by the quadrupole radiation formula of GW and hence the suggestion that $\omega_{\mathrm{i}} \propto M^{3} / R^{4}$ (Andersson \& Kokkotas 1998), we plot $\omega_{\mathrm{i}} I^{2} / M^{5}$ against $\eta^{2}$ (i.e., $M^{3} / I$ ) in Figure 2 and see that all data points now fall on a single straight line with high accuracy:

$$
I^{2} \omega_{\mathrm{i}} / M^{5}=-0.00694+0.0256 \eta^{2} .
$$

Therefore, we conclude that moment of inertia is a good physical parameter to unveil the universality inherent in the f-modes of compact stars.

To compare the accuracy of various proposed universal curves for the f-modes quantitatively, we evaluate the root-mean-square percentage error $\sigma$ defined by

$$
\sigma \equiv \sqrt{\frac{1}{N} \sum_{i}^{N}\left(1-\frac{\hat{y}_{i}}{y_{i}}\right)^{2}} .
$$

Here $N$ is the total number of f-mode data considered in the sample. $y_{i}$ corresponds to the exact value of the physical quantity $y$ (e.g., $M \omega_{\mathrm{r}}$ and $M \omega_{\mathrm{i}}$ ) of the $i$-th f-mode data. $\hat{y}_{i}$ is the approximated value of $y$ inferred from the universal curves discussed above. In Table 1 we show the percentage error $\sigma$ in $\omega_{\mathrm{r}}$ and $\omega_{\mathrm{i}}$ for the data obtained from different universal curves and different samples of f-mode data, namely those of (i) NS shown in Figure 1 $\left(\sigma_{1}\right)$; (ii) NS considered in Andersson \& Kokkotas (1998) $\left(\sigma_{2}\right)$; and (iii) QS shown in Figure 1 $\left(\sigma_{3}\right)$.

It is clearly shown in Table 1 that the universal curves (6) and (7) proposed in the present paper, which considers mass and moment of inertia as two crucial parameters characterizing f-mode pulsations of compact stars, work nicely with high precisions. For $\omega_{\mathrm{r}}$, the error is less than $1 \%$, while for $\omega_{i}$, the error is only about $1-2 \%$. In particular, such high degree of accuracy prevails for both NS and QS, whereas the universal curves using conventional compactness fail to yield satisfactory result for QS. Thus, under the assumption that the f-mode GW signals from a compact star could be detected, we can then infer the mass and moment of inertia of the star from its f-mode using the universal curves (6) and (7), without any prior knowledge of its EOS and composition. We shall establish a feasible inversion scheme to achieve such purpose in the following section.

Before introducing the inversion scheme, we perform an independent test here to show that equations (6) and (7) are robust and accurate for other commonly considered EOS. We consider the f-mode QNM of NS constructed with five modern EOS of nuclear matter, namely N1H1 (Balberg \& Gal 1997), BBB2 (Baldo et al. 1997), BPAL12 (Bombaci 1995), FPS (Pandharipande \& Ravenhall 1989) and SLy4 (Douchin \& Haensel 2001), which have not been used in the derivation of equations (6) and (7). The root-meansquare percentage errors $\sigma$ for these f-modes are just $0.25 \%$ and $0.95 \%$, respectively. This once again demonstrates the robustness of our finding reported here. 
TABLE 2

Percentage error of OUR InVersion SCheme

\begin{tabular}{|cc|ccc|}
\hline EOS & $M$ & $\delta M / M$ & $\delta R / R$ & $\delta I / I$ \\
\hline \hline AU & 0.8 & -0.056 & -2.6 & -0.031 \\
\hline AU & 1.0 & -0.13 & -0.67 & -0.16 \\
\hline AU & 1.6 & 4.0 & 2.1 & 6.9 \\
\hline APR1 & 0.8 & -0.066 & -5.1 & -0.23 \\
\hline APR1 & 1.2 & -0.21 & -1.6 & -0.19 \\
\hline APR1 & 1.6 & 0.50 & -0.11 & 0.78 \\
\hline \hline EOS A & 1.535 & 0.40 & -1.2 & 0.27 \\
\hline EOS A & 1.328 & 1.1 & -0.46 & 1.8 \\
\hline EOS B & 1.405 & -0.17 & -3.5 & 0.54 \\
\hline EOS B & 0.971 & 1.4 & -4.1 & 2.7 \\
\hline GM24 & 1.536 & -2.2 & -4.9 & -2.6 \\
\hline GM24 & 1.405 & 2.5 & -1.1 & 5.1 \\
\hline \hline QS1 & 0.8 & -0.096 & $18(0.53)$ & -0.18 \\
\hline QS1 & 1.4 & 0.93 & $12(1.3)$ & 1.3 \\
\hline QS2 & 1.0 & -0.17 & $17(0.72)$ & -0.3 \\
\hline QS2 & 1.6 & 1.6 & $11(1.7)$ & 2.5 \\
\hline
\end{tabular}

\section{INVERSION SCHEME}

Assuming that both $\omega_{\mathrm{r}}$ and $\omega_{\mathrm{i}}$ of the f-mode of a compact star have been obtained from GW observations, we can then solve the simultaneous equations (6) and (7) to determine the mass $M$ and the moment of inertia $I$ of the star.

Moreover, making use of equation (4) proposed by Lattimer \& Schutz (2005), we can estimate the radius $R$ with the input of $M$ and $I$. Since all these three equations hold only approximately, the inferred values of $M$, $R$ and $I$ are expected to deviate from the exact values by $\delta M, \delta R$, and $\delta I$. As shown in Table 1, percentage errors in $\omega_{\mathrm{r}}$ and $\omega_{\mathrm{i}}$ predicted by equations (6) and (7) are small and usually less than a few percent. It is reasonable to expect that the percentage errors $\delta M / M$ and $\delta I / I$ are also small and such inversion scheme can lead to accurate determination of the mass and the moment of inertia of the star.

To validate the scheme outlined above and test its accuracy, we inferred $M, R$ and $I$ numerically from $\omega_{\mathrm{r}}$ and $\omega_{\mathrm{i}}$ for three categories of compact stars, namely NS considered in Figure 1 (with AU and APR1 EOS), NS considered in Andersson \& Kokkotas (1998) (with A, B and GM24 EOS), and QS (QS1 and QS2). Table 2 shows the percentage errors in mass, radius and moment of inertia of our scheme for these stars. In general, the inversion scheme works very well for NS and all three physical quantities can be determined up to a few percent or better. Our scheme also reproduces accurate values of $M$ and $I$ for QS. However, the inferred value of $R$ is less satisfactory $(\sim 10-20 \%)$. This is understandable as the empirical relation (4) only works for NS, but not QS. If, however, there is other independent evidence (e.g., via the observed thermal X-ray emission) suggesting that the star might be a QS, we could then use equation (5) proposed by Beiger \& Haensel (2002) to infer a better value of $R$ from $M$ and $I$. The percentage errors $\delta R / R$ obtained in this way are enclosed by parentheses in Table 2 . We see that the percentage error in $R$ is decreased from about $10 \%$ to $1 \%$ level. In summary, we expect that the inversion scheme proposed in the present paper is applicable regardless of the nature of the compact star in consideration.

\section{CONCLUSION AND DISCUSSIONS}

In this paper we study the universality embedded in f-mode pulsations of compact stars and propose to use it to determine the physical parameters (including mass, radius and moment of inertia) of a compact star from which the f-mode gravitational wave signal is detected. We first establish a pair of empirical equations (6) and (17) that can predict the frequency and the damping rate of f-modes from $M$ and $I$ with good accuracy for both NS and QS. We then apply such discovery to develop an inversion scheme to infer the mass, radius and moment of inertia of a compact star from its f-mode. In particular, the scheme is shown to work well for both NS and QS.

The universality discovered in the present paper takes moment of inertia into the consideration, replacing the role of radius used in previous attempts (Andersson \& Kokkotas 1996, 1998, Benhar et al. 1999, 2004, Tsui \& Leung 2005b). It is worth noting that the radius of a star is sensitive to the low-density part of its EOS, whereas f-mode frequencies are expected to be dominated by dynamical behavior in the high-density regime. It is therefore physically appealing to use moment of inertia, which measures global mass distribution, to study f-mode oscillations. As clearly shown in Table 1, the replacement of $R$ with $I$ indeed leads to a much improved universal behavior. As it is arguable that the accuracy of the inversion scheme directly reflects the quality of the universal behavior upon which the scheme is based, the universality discovered in the present paper leads to an accurate inversion scheme.

Finally, it is worthy of mentioning that the first gravitational-wave search sensitive to the f-modes has recently been carried out by the LIGO detectors (Abbott et al. 2008). While the current detectors have not yet detected gravitational waves directly, it is plausible that the advanced LIGO detectors, which have a factor of 10 improvement on the sensitivity, might detect the f-mode gravitational wave signals from compact stars. The inversion scheme proposed in this work will provide a unifying way to infer the mass, radius and moment of inertia of both NS and QS accurately. Of course in reality the situation is often complicated by the presence of magnetic field and rotation, in the future we shall 
consider the impacts of these factors on our proposal.

This work is supported in part by the Hong Kong Research Grants Council (Grant No: 401807) and the direct grant (Project ID: 2060330) from the Chinese University of Hong Kong. We thank J. Wu, Y. J. Zhang and P. O. Chan for helpful discussions and assistance.

\section{REFERENCES}

Abbott, B. et al. 2008, Phys. Rev. Lett., 101, 211102

-. 2009a, Phys. Rev. D., 80, 062001

- 2009b, Phys. Rev. D., 80, 047101

—. 2009c, arXiv:0909.3583 [astro-ph.HE]

Akmal, A., Pandharipande, V. R., \& Ravenhall, D. G. 1998, Phys. Rev. C, 58, 1804

Andersson, N. \& Kokkotas, K. D. 1996, Phys. Rev. Lett, 77, 20

. 1998, MNRAS, 299, 1059

Baade, W. \& Zwicky, F. 1934, Phys. Rev., 45, 138

Balberg, S. \& Gal, A. 1997, Nucl. Phys. A, 625, 435

Baldo, M., Bombaci, I., \& Burgio, G. F. 1997, A\&A, 328, 274

Bejger, M., Bulik, T., \& Haensel, P. 2005, MNRAS, 364, 635

Bejger, M. \& Haensel, P. 2002, A\&A, 396, 917

Benhar, O., Berti, E., \& Ferrari, V. 1999, MNRAS, 310, 797

Benhar, O., Ferrari, V., \& Gualtieri, L. 2004, Phys. Rev. D, 70 124015

Bodmer, A. R. 1971, Phys. Rev. D., 4, 1601

Bombaci, I. 1995, Perspectives on Theoretical Nuclear Physics, ed. by I. Bombaci, A. Bonaccorso, A. Fabrocini, et al. (Pisa: Edizioni ETS), 223

Canuto, V. \& Chitre, S. M. 1974, Phys. Rev. D, 9, 1587

Chandrasekhar, S. \& Ferrari, V. 1991, Proc. R. Soc. A, 432, 247

Ching, E. S. C., Leung, P. T., van den Brink, A. M., Suen, W. M., Tong, S. S., \& Young, K. 1998, Rev. Mod. Phys., 70, 1545

Cohen, J. M., Langer, W. D., Rosen, L. C., \& Cameron, A. G. W. 1970, Ap\&SS, 6, 228

Detweiler, S. \& Lindblom, L. 1985, ApJ, 292, 12

Douchin, F. \& Haensel, P. 2001, A\&A, 380, 151

Glendenning, N. K. 1997, Compact Stars - Nuclear Physics, Particle Physics, and General Relativity (Springer, NY)
Haensel, P., Potekhin, A. Y., \& Yakovlev, D. G. 2007, Neutron stars 1: Equation of state and structure, Astrophysics and space science library, Vol. 326 (New York: Springer)

Itoh, N. 1970, Prog. Theor. Phys., 44, 291

Kokkotas, K. D., Apostolatos, T. A., \& Andersson, N. 2001 MNRAS, 320, 307

Kokkotas, K. D. \& Schmidt, B. G. 1999, Living Rev. Rel., 2, 2

Lattimer, J. \& Prakash, M. 2007, Phys. Rep., 442, 109

Lattimer, J. M. \& Prakash, M. 2001, ApJ, 550, 426

Lattimer J. M. \& Schutz, B. F 2005, ApJ, 629, 979

Leaver, E. W. 1986, Phys. Rev. D, 34, 384

Lindblom, L. \& Detweiler, S. L. 1983, ApJ, 53, 73

Pandharipande, V. 1971a, Nucl. Phys A, 174, 641

—. 1971b, Nucl. Phys A, 178, 123

Pandharipande, V. R. \& Ravenhall, D. G. 1989, Proc. NATO Advanced Research Workshop on nuclear matter and heavy ion collisions, Les Houches, ed.M. Soyeur et al. (Plenum, New York), 103

Press, W. 1971, ApJ, 170, L105

Sotani, H. \& Harada, T. 2003, Phys. Rev. D, 68, 024019

Sotani, H., Kohri, K., \& Harada, T. 2004, Phys. Rev. D, 69, 084008

Thorne, K. S. \& Campolattaro, A. 1967, ApJ, 149, 591

Tsui, L. K. \& Leung, P. T. 2005a, Phys. Rev. Lett., 95, 151101 . 2005b, MNRAS, 357, 1029

Wiringa, R. B., Fiks, V., \& Fabrocini, A. 1988, Phys. Rev. C, 38, 1010

Witten, E. 1984, Phys. Rev. D, 30, 272

Wong, K. S., Lin, L. M., \& Leung, P. T. 2009, ApJ, 699, 1809 